\title{
Weighting in the dark: resource allocation in the new NHS
}

\author{
Trevor A Sheldon, George Davey Smith, Gwyn Bevan
}

National allocation of resources to regional health authorities and by them to districts is now determined by a weighted capitation formula. The national formula was derived from regression analysis, with hospital utilisation as an index of need for health care-a method which has fundamental limitations. This paper argues that the search for an empirically based resource allocation formula of high precision in the name of promotion of equity is largely fruitless given the impossibility of measuring the true need for, and costs of, providing health care, especially with the limited data available. The inclusion of measures of social deprivation is also poorly thought out. The availability of data from the 1991 census, which included a question regarding longstanding illness, together with the intention of the Department of Health to review the weighted capitation formula using this information may stimulate much work but little light. It is essential that the impact of resource allocation formulas is justifiable on grounds other than the composition of any particular formula.

\section{Introduction}

Since the report of the Resource Allocation Working Party (RAWP) ${ }^{1}$ revenue allocations of regional health authorities for hospital and community health services have been moved towards targets derived from a weighted capitation formula. The principles laid out by RAWP underlay regional targets until 1991, when a new formula was used. ${ }^{2}$ The Department of Health is also keen that this formula should be used as the basis for subregional distribution. As these resources affect the "purchasing power" of the commissioning authorities the use of the formula is seen as a way of creating a level playing field for purchasers.

Resource allocation mechanisms are always contentious but because of reduced real growth in total funding in the current year debate over the way resources are shared between and within regions has intensified. The National Health Service Management Executive is about to review the weighted capitation formula in the light of the 1991 census data. Any change is likely to set the framework for the distribution of NHS finances for the foreseeable future. It is crucial now to consider the fundamental issues which analysis ought to address. Our concern is that the availability of data from the new census, together with the increase in access to computers since the last census, is likely to lead to a veritable orgy of statistical analysis, and, if the past is a reliable guide to the future, this analysis will obscure rather than illuminate fundamental issues in resource allocation. This paper aims to explore some of the key issues, clearly set out some of the pitfalls, and provoke some deeper thought and debate about how new research can usefully inform resource allocation.

In particular, this paper will highlight some of the tensions between the empirical method of producing resource allocations based on statistical modelling of the available data and a more normative approach which allocates according to a more explicit theoretical rationale and political choices.

\section{Effects of the new formula}

There is much confusion within health authorities about the construction of the new formula which weights the age adjusted population of each region by the square root of its under 75 years, all cause, standardised mortality ratio. The formula is derived from the review of RAWP, ${ }^{3}$ which carried out a detailed statistical regression analysis using ward based hospital utilisation data from six regions. ${ }^{4}$ Though the RAWP review made some sensible changes to the national formula such as using all cause premature standardised mortality ratio, the resulting weighting was particularly contentious.

In the original RAWP formula ${ }^{1}$ standardised mortality ratio was included with a weighting (and elasticity) of 1 -that is, a $10 \%$ increase in standardised mortality ratio increased the weighting of that region, and hence its revenue target, by $10 \%$. The review of RAWP estimated an equation in which standardised mortality ratio has an elasticity of roughly 0.5 , so the same $10 \%$ increase in standardised mortality ratio would lead to only a $5 \%$ increase in funding target. ${ }^{4}$ (As the equation used a logarithmic scale a coefficient of 0.5 is the equivalent of the square root of standardised mortality ratio in the unlogged formula, which explains the use of the square root of standardised mortality ratio in the national weighted capitation formula.) The reduced weighting of standardised mortality ratio in the final formula resulted in a shift in resources from the regions with higher premature mortality (predominantly in the north) to those with lower premature mortality (mainly in the south east) ${ }^{5}$ as shown in the first column of the table. The large amounts of money involved mean that, though small, the percentage shifts have a considerable effect on resources received by each region. The review of RAWP also examined the use of census based socioeconomic variables (so called "social deprivation" factors) to explain variation in health service use over and above that associated with the standardised mortality ratio. However, despite the fact that various combinations of social variables were statistically significant predictors of utilisation ${ }^{4}$ none were included in the new weighted capitation formula. ${ }^{2}$

The Department of Health decided to adopt part of the model (the reduced weighting for standardised mortality ratio) and ignore the index of social factors. This pushed most regions further from their original RAWP targets than the full model would have done and increased the relative shift of resources to the south east. The table shows how the funding targets of the 14 English regions are affected by the use of the reduced weighting of standardised mortality ratio and the effect that including the socioeconomic variables would have
Correspondence to: Mr Sheldon.

BMF 1993;306:835-9 
Effects of weighted capitation formula on regional targets compared with RAWP targets of same year

\begin{tabular}{lcc}
\hline & $\begin{array}{c}\text { \% Change when using } \\
\text { weighted under 75 years } \\
\text { standardised mortality } \\
\text { ratio }\end{array}$ & $\begin{array}{c}\text { \% Change when using } \\
\text { weighted under 75 years } \\
\text { standardised mortality } \\
\text { ratio and social } \\
\text { deprivation }\end{array}$ \\
Region & -2.6 & -0.9 \\
\hline North Western & -0.6 & 0.7 \\
Yorkshire & -1.3 & -0.3 \\
Northern & -1.1 & -0.5 \\
Mersey & 1.6 & -0.2 \\
South West Thames & 0.1 & -1.5 \\
Oxford & 2.8 & 1.4 \\
Wessex & 1.9 & 0.5 \\
South Western & 1.9 & 1.7 \\
North West Thames & -1.7 & -1.7 \\
West Midlands & 0.8 & 1.9 \\
North East Thames & 1.1 & 1.7 \\
South East Thames & -0.8 & -1.6 \\
Trent & -0.1 & -1.0 \\
East Anglia & Source: adapted from Royston et al. ${ }^{10}$ &
\end{tabular}

had. In the first nine regions, including the social variables would have resulted in a change of target allocations which opposed the effect of reducing the weighting of standarised mortality ratio-that is, they act in the same direction as standardised mortality ratio. In seven of these regions failure to include the social variables resulted in a reduction of target revenue of between $1 \cdot 0 \%$ and $1 \cdot 8 \%$.

\section{Problems with the new formula}

EMPIRICAL MODEL

There has been criticism of the methodological validity and statistical soundness of the review of RAWP. ${ }^{6-8}$ One of the aims of the review was to produce a formula which was empirically based as opposed to the original RAWP formula, which included standardised mortality ratio as a proxy for need on theoretical grounds. This empirical emphasis is only sensible when the factors which policy makers think merit funding can be measured accurately enough. As resource allocation is meant to embody the principle of equal access for equal need this presents the problem of measuring need for health care. This concept may be classed as one which is "essentially contested"-that is, a concept whose application is inherently a matter of dispute.

\section{BASED ON UTILISATION}

Utilisation measured by admission rates at the small area level was used as the measure of need, with an attempt to adjust for the current level of supply of health care facilities. ${ }^{40}$ This raises two important questions. Firstly, as there is consistent evidence of variations in use of services being explained by variations in supply, a formula based on variations in use between even small areas will partly reflect variations in supply. ${ }^{611}$ Using measures which explain variations in utilisation in normative formulas is to confuse what is with what ought to be. The reductio ad absurdum is a formula which funds the status quo through specious "objective" measures. Secondly, the objective of the weighted capitation formula is not to identify relative need or morbidity but to allocate resources with the aim of meeting these needs. It is then also necessary to estimate the costs of treating that morbidity, ${ }^{7}$ something which neither RAWP nor the review attempted. The fact that one area has $30 \%$ greater utilisation does not imply $30 \%$ more cost if the pattern of morbidity (case mix) is different. But it is difficult to see how normative assessments of these variations can be made.

\section{INSTABILITY}

The weighted capitation formula has been advanced not only as a mechanism for allocating national resources among the 14 regions but also for subregional allocation. Given that it is based on a model which examined variation among electoral wards within only a few regions it could be argued that it is more validly applied at a local level. ${ }^{8}$

However, the model is unstable in that though the average weighting of standardised mortality ratio was around $0.5(0.44)$ for the six regions, it ranged from 0.2 in Wessex to 0.72 for the Trent region, depending on which regional dataset is used. ${ }^{4}$ The coefficients are likely to vary even more widely for those regions whose data were not included in the modelling exercise.

Empirical methods are fraught with problems. They certainly provide no good evidence for reducing the weight given to standardised mortality ratios in the formula and there is no justification for encouraging the use of the national formula for subregional allocation. As a result of the general confusion and criticisms of the national formula regions have adopted a wide range of different formulas for subregional allocation, ${ }^{12}$ often commissioning local research, some of which repeats the errors of the RAWP review.

\section{Social deprivation}

There has been considerable pressure to incorporate a measure of social deprivation in resource allocation formulas, ${ }^{1314}$ and several regions have already included some form of deprivation weighting in their subregional allocation. ${ }^{12}$ The rationale behind this is not always clear. A common justification is that social deprivation is a cause of (or associated with) morbidity. ${ }^{15}$ However, because deprivation is also associated with excess mortality some of this is already picked up by including standardised mortality ratio. Though there is some additional variation in utilisation rates which is explained by social factors, it is relatively small ${ }^{4}$ and does not necessarily mean that additional variation in morbidity would be explained.

Another justification advanced is that social deprivation variables tap aspects of need for resources not adequately represented by morbidity measures-for example, that it is more difficult to provide services in a deprived area or that effectiveness is reduced by adverse conditions. However, rarely are such rationales clearly worked out or attempts made to provide evidence in support of these claims. In addition, it is claimed that people of different socioeconomic groups make different demands on the health service at similar levels of sickness or "objective" need and that the cost of meeting these needs may also vary. This, however, is mostly supposition and there is little evidence to support these arguments or to indicate the resource implications of this even if they are valid.

\section{"Using measures which explain variations in utilisation in normative formulas is to confuse what is with what ought to be."}

Even if true, there is little evidence to show that the bulk of hospital care is particularly sensitive to meeting the needs of deprived populations.

Several studies have recorded socioeconomic differences in the uptake of various forms of health care by individuals using data from the general household survey $^{16}$ and the third national morbidity survey. Weights have also been derived by using self reported chronic sickness as a measure of morbidity and a proxy for health care need. However, these relations are likely to be geographically unstable. Recent work has shown that the strong correlation between scores on 
the Nottingham health profile and deprivation scores for the ward of residence for a sample of people in London $^{18}$ was not reproducible in non-metropolitan areas. $^{19}$

There is little justification in using weights derived from individually based analyses in a regional formula ${ }^{20}$ for two main reasons. Firstly, because they are derived without adjustment for standardised mortality ratio their use in a formula containing standardised mortality ratio will result in considerable double counting. Secondly, as the weights are derived from analysis of individuals there is a serious problem of applying them at the level of wards or districts where the information is readily available only in aggregate. This can result in a significant mis-estimation of the resource needs of the area.

"BEST" INDEX OF DEPRIVATION

Several other deprivation indices have been advanced and are used elsewhere. ${ }^{122122}$ However, the choice significantly affects the final geographical distribution ${ }^{43}$ and their use in resource allocation has been questioned. ${ }^{24} 25$ There is an evolving industry

\section{"Commissioning research on the use of deprivation indices ... is ... seen as a means of arguing ... for a bigger share."}

where indices are developed which measure deprivation more accurately and attempt to identify the "best" one. ${ }^{26}$ These indices are usually validated against utilisation rates (see above) or by how well they correlate with various measures of morbidity available routinely. Such efforts are, we believe, misdirected. If it is decided that use of a measure of material deprivation is informative and conceptually sound, then simple measures based on easily available and regularly updated measures would be preferable to the opaque and statistically complex derived indices. Data such as unemployment rates have been shown to perform as well as other, more complex indices ${ }^{27}$ and are likely to have a more stable social meaning across areas and over time.

This raises the question of why measures of deprivation are being put forward instead of morbidity measures. The lack of a gold standard for health service need was a reason for using standardised mortality ratio in the RAWP formula. ${ }^{13}$ The decision to use measures of deprivation as a proxy for need seems to sidestep the problem, with debate revolving around how well the proxy-deprivation-can be measured. Indeed, discussions of this aspect often seem to consider the function of health services to be to compensate for deprivation itself rather than for its health damaging effects. Even using apparently direct measures of morbidity is problematical, as reporting may be different between social groups, especially in response to general questions such as the "limiting longstanding sickness" item in the general household survey and 1991 census. $^{28}$ The pattern of appropriate allocation is also highly sensitive to the morbidity measures used and it is not clear how variations in morbidity relate to actual resource needs. ${ }^{29}$

Those health authorities which express most interest in refining formulas by varying the factors included tend to be those which have lost out with the current formula. Commissioning research on the use of deprivation indices in allocating formulas is therefore often seen as a means of arguing more effectively for a bigger share. It is difficult for conceptually sound research to be conducted in such an environment.

In short, those who propose a deprivation weighting must decide whether this is to be additional to or a substitute for mortality. If an indicator of social deprivation is additional to mortality, then how are the effects of each to be identified and understood? We have described the problems of using data on utilisation of services which we see as offering no reliable way of weighting different indicators. If an indicator of social deprivation is a substitute for mortality, then this means discarding the best available direct proxy for morbidity, which is after all what much of the NHS seeks to respond to rather than some ill defined concept of social deprivation.

\section{Case for standardised mortality ratios and age weighting}

Some regional health authorities have sought to abandon the use of standardised mortality ratios altogether. One argument advanced for this is that it makes no sense to include mortality data in a formula for resource allocation as dead people make no use of health services. To this there are two responses. Firstly, standardised mortality ratios broadly reflect the cumulative morbidity and social experience of an area and provide more stable and comprehensive measures of morbidity than utilisation rates (or, indeed, contemporaneous deprivation indices). It has been well established that death rates are highly correlated with the incidence of chronic diseases which are known to justify health service intervention. ${ }^{13}$ Secondly, as Knox argued after publication of the RAWP report, ${ }^{30}$ people who are dying are among the heaviest users of services. A famous American study indicated that as much as $28 \%$ of Medicare expenditure was spent on those in the last year of life. ${ }^{31}$ In the United Kingdom it is not possible to get comparative estimates for individuals, but in England and Wales in $1984,23 \%$ of non-psychiatric beds were occupied by people who died before discharge (G Bevan, unpublished observations). Hence variations in mortality not only indicate variations in morbidity but also variations in the great need for services in caring for those ith conditions with a high number of deaths.

Thus an important contribution to the age weighting in the formula which uses ae related utilisation at national average rates is due to the utilisation of very elderly people who subsequently die. ${ }^{32}$ It is therefore crucial to include standardised mortality ratios alongside age weightings to correct for variations in life expectancy. A district with a high under 75 years standardised mortality ratio will tend to have people dying earlier than expected in the younger age groups, and applying national average rates of use of service to those groups will underestimate its relative need for health services.

Using utilisation data to derive age weights suffers from similar problems to those outlined for weighting standardised mortality ratio, though they are at least derived from individual as opposed to area based data and so are not subject to the ecological fallacy. The national data on use of services by age may indeed not truly reflect needs of individuals at different ages; there is, after all, evidence of rationing by age, so the elderly may not get the health care they need. But this raises ethical as well as clinical judgments. There is a natural appeal in using age weighting in the formula based on the normative assumption that individuals in a given age group ought to have the same utilisation of services wherever they live. It is hard to see what sense there is in, for example, assuming that individuals without a car ought to have the same utilisation of services wherever they live. This is true for any social variables 
including social class which are not necessarily geographically robust proxies for exposure to risk factors and for health care needs. The issue of age weighting does, however, highlight a problem with migration of elderly people to retirement areas, which are then given a relatively high level of estimated need for health care resources. Variations in need in the elderly are a particular problem for which there is currently no satisfactory indicator. This requires further investigation.

\section{The future: what value another review?}

The Department of Health has signalled its intention to review the formula in the light of 1991 census data, again using small area analysis. As by the time any new formula is developed and implemented the information will be nearly as out of date as that used in the RAWP review, any new analysis may be little more informative than the last with respect to the role of social variables.

Much attention will focus on the inclusion in the census for the first time of the self reported limiting, longstanding sickness item. However, although the availability of new and more contemporary data generates much activity, interest, and publishable analyses, it might not cast much more light on the resource allocation problem for the reasons outlined above. As all cause standardised mortality ratio is highly correlated with, and so is a good proxy for, chronic sickness ${ }^{13}$ it is not clear how much extra information the new census question will provide for mapping the distribution of health care need useful for resource allocation. There is also, of course, a new problem with the 1991 census, of underrecording probably because of the poll tax. This threatens the reliability of both basic population data and attempts to use these new census data in social indicators to identify poverty.

\section{"By the time any new formula is... implemented the information will be... out of date."}

More research using the new data can be useful, however, in exploring the sensitivity of the model to a variety of changes in assumptions and included factors. For example, though the use of under 75 years standardised mortality ratio is more sensitive to regional variations, any measure of death rates with an absolute age cut off will be dominated by the mortality experience of men who have a shorter average life expectancy. In order to compensate for this it would be worth exploring the effects of using, say, under 65 years standardised mortality ratio for men and under 75 years standardised mortality ratio for women, which will be based on similar numbers of deaths. Other measures of mortality experience, such as years of potential life lost, which have different age weightings and are more sensitive to social class differentials, ${ }^{33}$ are also worth exploring. ${ }^{34}$ It is also worth examining how mortality indices best combine with age weighting to reflect relative needs for health care. Knox, ${ }^{30}$ for example, showed how the original RAWP formula essentially allocated resources according to crude death rates.

\section{INEVITABLE MOVE TO UNVALIDATABLE PROXIES}

However, it must be recognised that a comprehensive and accurate empirically bàsed resource allocation formula cannot be achieved. This is because once the decision has been made to move away from incremental allocation based on historic spending the question of what merits funding is raised before issues of measurement. This cannot be articulated in a sufficiently quantifiable form, and even those elements more amenable to measurement will not be able to be measured accurately. Inevitably we are moved in the direction of using proxies which cannot properly be validated.

Once the use of a partial empirical model is accepted then it will be open to constant revisions and adjustments as new data become available. It will also become the focus of political lobbying by health authorities who stand to gain by this or that factor being included. The resulting contention and potential instability will hinder long term health care planning. This is made worse by the lack of clear objectives for the health service to which the formula can respond and attempt to follow.

As long as the limitations are recognised and understood and those who allocate are seen to be acting fairly and using socially accepted criteria such as equity and efficiency, ${ }^{7}$ the use of rough, though conceptually sound, mechanisms (for example, the original RAWP formula) is most probably as good as we can get. There are real limits to the use of statistical modelling in this area for two reasons. Many of the factors (social or otherwise) which may affect either the need for or the cost of delivering health care are unevenly distributed, often in small pockets, and do not present in the same way-or have the same social meaning-in different parts of Britain. These may take the form of problems which even if proved empirically to influence resource needs do so in such particular, local, and sometimes subtle ways that statistical models are unlikely to be sensitive enough to be helpful. Modelling will tend to mask small but important variations which may be adequately understood only by local research.

For example, it has been shown in certain studies that providing health care to some more isolated rural populations is more expensive than in less sparsely populated areas. ${ }^{35}$ However, it is impossible to produce some rural weighting which is generalisable to all rural areas. Similarly the extra health care needs of homeless people which have been documented in parts of London $^{36}$ reflect local conditions and cannot validly be applied to all areas. More research needs to be done to attempt to understand these problems but statistical modelling is not appropriate.

It is not possible to measure accurately the cost of meeting health care needs, the magnitude of health care needs, or their proxies, and then proxies of proxies, down the line. Therefore complex data derived methods which obscure rather than highlight the provisional nature or sensitivity of the models should not be developed.

\section{ACCOUNTABILITY OF COMMISSIONING AUTHORITIES}

As districts merge and form larger purchasing organisations and the overall variability between health authorities is reduced due to averaging, so increasing amounts of the variation will be contained within authorities and therefore beyond the reach of resource allocation formulas despite the devolution reflected in locality planning. This should focus our attention more on the nature of health care commissioning activity. What mechanisms are in place to ensure that health care services are purchased in a manner likely to result in equity between social groups and individuals? This is an important issue which needs addressing given that commissioning authorities are not particularly accountable to the public and that an increasing proportion of purchasing power has devolved to general practitioner fundholders, who are not neces- 
sarily geographically distributed in proportion to need. The problems of developing a capitation formula for fundholders raises, of course, all the problems discussed here with two further complications: the small numbers of practice populations and the potential selection of patients by practices (cream skimming).

Public health physicians propose the technology of health needs assessment as the mechanism by which the purchasing process will be made to respond to the distribution of the population's needs. However, there is little basis for confidence, since, as Frankel writes, "research activity has been concerned almost exclusively with the probability of neediness and not with the distribution of those who might be expected to benefit from particular interventions. The established measures of need are ... of uncertain relevance to the decisions about the need for health care that concern those engaged in commissioning provision." ${ }^{37}$

\section{"Authorities can gain only at the expense of others."}

The preoccupation in the past has been with the total size of the health service budget, how it is shared out at the macro level, and how much activity it funds. As the use of equity based financing mechanisms reduces the degree of inequality of allocation nationally the potential benefits to be derived from developing increasingly elaborate models for allocation are likely to be small. Attention should increasingly be focused on how resources are used-the effectiveness and appropriateness of health service interventions ${ }^{3839}$-studying the distribution of indications for health care as a means of increasing not only efficiency but also equity within the context of democratically driven priorities.

The purpose of this paper has been to argue against weighting in the dark: an industry of resource allocation which is abstracted from research into and the management and delivery of health services. As resource allocation is a "zero sum game" (that is, authorities can gain only at the expense of others) it seems essential to be able to explain why different formulas produce different results in terms other than the composition of the formulas. The common failure to produce such explanations threatens to undermine the clear principles of resource allocation as stated in the original RAWP report and to bring NHS formulas into disrepute.

We do see scope for research into resource allocation but believe this ought to be quite different from the common practice of the 1980 s of relating census variables to utilisation rates. The new kinds of research we advocate would aim to produce clearer understanding of variations in need for and utilisation of health services, with the objective of identifying how resources can be used more equitably and efficiently. These after all were stated in the original RAWP's terms of reference and remain as valid now as they were in 1975.
1 Department of Health and Social Security. Sharing resources for health in England. London: DHSS, 1976. (Report of the Resource Allocation Working Party.)

2 Department of Health. Funding and contracts for health services. Working paper 2. London: HMSO, 1989.

3 Department of Health and Social Security. Review of the Resource Allocation Working Party formula: final repon by the NHS Management Board. London: DHSS, 1988.

4 Coopers and Lybrand. Integrated analysis for the review of RAWP. London: Coopers and Lybrand, 1988.

5 Leck I. The north-south divide in England: implications for health care resource allocation. Community Med 1989;11:102-7.

6 Mays N. NHS resource allocation after the 1989 white paper: a critique of the research for the RAWP review. Community Med 1989;11:173-86.

7 Carr-Hill R, Sheldon TA. Rationality and the use of formulae in the allocation of resources to health care. I Public Health Med 1992;14:117-26.

8 Sheldon TA, Carr-Hill R. Resource allocation by regression in the National Health Service: a critique of the Resource Allocation Working Party's Health Service: a critique of the Resource Allocation Working
review. foumal of the Royal Statistical Society $(A)$ 1992;155:403-20.

9 Gallie WB. Essentially contested concepts. Proceedings of the Aristotlian Society 1955-6;56:167-98.

10 Royston GHD, Hurst JW, Lister EG, Stewart PA. Modelling the use of health services by populations of small area to inform the allocation of central resources to larger regions. Socio-economic Planning Sciences 1992;26: 169-80.

11 Morgan M, Mays N, Holland WW. Can hospital use be a measure of need for health care? I Epidemiol Community Health 1987;41:269-74.

12 National Association of Health Authorities and Trusts. Weighted capitation survey. Birmingham: NAHAT, 1992.

13 Mays N, Bevan G. Resource allocation in the health service. London: Bedford Square Press, 1987. (Occasional Papers on Social Administration, No 81.)

4 Moore W. Cash allocation is "unfair" to socially deprived districts. Health Service fournal 1992;102:6.

15 Townsend P. Deprivation and ill health: new scientific evidence and the implications for policy. Southampton: Institute for Health Policy Studies, 1990

16 Balarajan R, Yuen P, Machin D. Socioeconomic differentials in the uptake of medical care in Great Britain. I Epidemiol Community Health 1987;41:196-9.

17 Ben Shlomo Y, White I, McKeigue PM. Prediction of general practitioner workload for census based social deprivation scores. $\mathcal{f}$ Epidemiol Community Health 1992;46:532-6.

18 Curtis SE. Use of survey data and small area statistics to assess the link between individual morbidity and neighbourhood deprivation. If Epidemiol Community Health 1990;44:62-8.

19 Jessop EG. Individual morbidity and neighbourhood deprivation in a nonmetropolitan area. $\mathcal{F}$ Epidemiol Community Health 1992;46:543-6.

20 Balarajan R. Social deprivation and age adjustment ratios for SW Thames region. Guildford: Epidemiology and Public Health Research Unit, University of Guildford: Epidey, 1990 .

21 Carstairs V, Morris R. Deprivation, mortality and resource allocation Community Med 1989;11:364-72.

22 Jarman B. Social deprivation and health service funding. London: Imperial Coilege of Science, Technology, and Medicine, University of London, 1990 (Papers in Science, Technology, Medicine and Public Policy, No 22.)

23 Hutchinson A, Foy C, Sandhu B. Comparison of two scores for allocating resources to doctors in deprived areas. $B M \mathcal{F}$ 1989;299:1142-4.

24 Davey Smith G. Second thoughts on the Jarman index. BMF 1991;302:359-60.

25 Carr-Hill RA, Sheldon TA, Thunhurst C. Politics of deprivation indices. In: Scott N, ed. Deprivation indices and health. Warwickshire: University of Warwick. In press.

26 Morris R, Carstairs V. Which deprivation? A comparison of selected deprivation indices. F Public Health Med 1991;13:318-26.

27 Campbell DA, Radford JMC, Burton P. Unemployment rates: an alternative to the Jarman index? $B M$ F 1991:303:750-5.

28 O'Donnell O, Propper C. Equity and the distribution of UK National Health Service resources. Fournal of Health Economics 1991;10:1-19.

29 Carr-Hill RA, Maynard AK, Slack R. Morbidity variation and RAWP $f$ Epidemiol Community Health 1990;44:271-3.

30 Knox EG. Principles of allocation of health care resources. I Epidemiol Community Health 1978;32:3-9.

1 Lubitz J, Prihoda R. The use and costs of health care in the last two years of life. Health Care Financing Review 1982;5:117-31.

32 Fuchs VR. Though much is taken: reflections on aging, health and medical care. Milbank Memorial Fund Quarterly 1984;62:143-66.

33 Blane D, Davey Smith G, Bartley M. Social class differences in years of potential life lost: size, trends and principal causes. $B M \mathcal{F}$ 1990;301:429-32.

34 Palmer S, West $\mathrm{P}$, Patrick D, Glynn M. Mortality indices in resource allocation. Community Med 1979;1:275-81.

35 Watt I, Franks A, Sheldon TA. Health and health care in rural areas. BMF 1993. (In press.)

36 Victor $C$. Health status of the temporarily homeless population and residents of North West Thames region. BMF 1992;305:387-91.

37 Frankel S. The epidemiology of indications. I Epidemiol Community Health 1991;45:257-9.

38 Tudor Hart J. Two paths for medical practice. Lancet 1992;340:772-5.

39 Long AF, Sheldon TA. Effectiveness bulletins: enhancing effective and acceptable purchaser and provider decisions: overview and methods. Quality in Health Care 1992;1:74-6.

(Accepted 21 fanuary 1993) 